\title{
The Power Integrations of Trigonometric and Hyperbolic Functions
}

\author{
Ahmed M. Ali*, Omar M. Abd-Alkanee \\ The Surveying Department, Department of Mathematics, University of Mosul, Mosul, Iraq \\ Email: *ahmed_math79@yahoo.com,moammed.omar8875@yahoo.com
}

How to cite this paper: Ali, A.M. and Abd-Alkanee, O.M. (2019) The Power Integrations of Trigonometric and Hyperbolic Functions. Open Access Library Journal, 6: e5618.

https://doi.org/10.4236/oalib.1105618

Received: July 19, 2019

Accepted: August 26, 2019

Published: August 29, 2019

Copyright $\odot 2019$ by author(s) and Open Access Library Inc.

This work is licensed under the Creative Commons Attribution International License (CC BY 4.0).

http://creativecommons.org/licenses/by/4.0/ (c) (i) Open Access

\begin{abstract}
For importance of the trigonometric integrals, we have in this paper finding a series of power, some of trigonometric functions that did not exist before in the first section. As shown in the Section two, where, the integration of trigonometric function with power $\mathrm{n}$ has been achieved and approved, this result is considered as the first achievement. while in the third section we find integrals for multiplying of trigonometric functions with powers $\mathrm{n}$ and $\mathrm{m}$. Finally, in Section 4, we find series of power of hyperbolic functions, integrals of hyperbolic functions with powers $\mathrm{n}$ and integrals for multiplying of hyperbolic functions with powers $\mathrm{n}$ and $\mathrm{m}$.
\end{abstract}

\section{Subject Areas}

Financial Mathematics, Function Theory, Functional Analysis, Integral Equation

\section{Keywords}

Trigonometric Functions, Hyperbolic Functions, Integrations Method

\section{Introduction}

We follow the terminology of [1] [2] [3]. The Trigonometry had resulted from the continuous interaction between mathematics and astronomy, and this remained the special relationship between trigonometry and astronomy until the third century AD, when it began to disconnect Nasir al-Din al-Tusi (1201-1274) AD. In the middle of the seventeenth century when calculus has been developed by Issac Newton and that is done by inventing new form and relationship between mathematics and physical phenomena, moreover. Newton's work proved many functions as a series of infinity. with respect $x$ and then Newton obtained series since $x$ and similar series of cosine of $x$ as well as tangent $x$ with the inven- 
tion of calculus, and re-considered the trigonometric functions where still play an important role in both pure and applied mathematics analysis [4] [5].

Theorem 1.1 [6]: For all $k \in N$, then:

1) $\sin ^{2 k-1}(x)=\frac{(-1)^{k-1}}{2^{2 k-2}} \sum_{n=1}^{k}(-1)^{n-1}\left(\begin{array}{c}2 k-1 \\ n-1\end{array}\right) \sin (2 k-2 n+1) x$.

2) $\sin ^{2 k}(x)=\frac{1}{2^{2 k}}\left(\begin{array}{c}2 k \\ k\end{array}\right)+\frac{(-1)^{k}}{2^{2 k-1}} \sum_{n=0}^{k-1}(-1)^{n}\left(\begin{array}{c}2 k \\ n\end{array}\right) \cos (2 k-2 n) x$.

3) $\cos ^{2 k-1}(x)=\frac{1}{2^{2 k-2}} \sum_{n=1}^{k}\left(\begin{array}{c}2 k-1 \\ n-1\end{array}\right) \cos (2 k-2 n+1) x$.

4) $\cos ^{2 k}(x)=\frac{1}{2^{2 k}}\left(\begin{array}{c}2 k \\ k\end{array}\right)+\frac{1}{2^{2 k-1}} \sum_{n=0}^{k-1}\left(\begin{array}{c}2 k \\ n\end{array}\right) \cos (2 k-2 n) x . \#$

Theorem 1.2: For all $k \in N$, then:

$$
\begin{gathered}
\tan ^{2 k}(x)=(-1)^{k}+\sec ^{2}(x) \sum_{n=1}^{k}(-1)^{n+1} \tan ^{2 k-2 n}(x) \\
\tan ^{2 k+1}(x)=(-1)^{k} \tan (x)+\sec ^{2}(x) \sum_{n=1}^{k}(-1)^{n+1} \tan ^{2 k-2 n+1}(x) \\
\cot ^{2 k}(x)=(-1)^{k}+\csc ^{2}(x) \sum_{n=1}^{k}(-1)^{n+1} \cot ^{2 k-2 n}(x) \\
\cot ^{2 k+1}(x)=(-1)^{k} \cot (x)+\csc ^{2}(x) \sum_{n=1}^{k}(-1)^{n+1} \cot ^{2 k-2 n+1}(x)
\end{gathered}
$$

\section{Proof:}

1) We use mathematical induction on $k, k \geq 1$.

For $k=1$, we find by direct:

$$
\tan ^{2}(x)=-1+\sec ^{2}(x) .
$$

For $k=2$, then

$$
\begin{aligned}
\tan ^{4}(x) & =\tan ^{2}(x)\left(-1+\sec ^{2}(x)\right) \\
& =1-\sec ^{2}(x)+\tan ^{2}(x) \sec ^{2}(x) \\
& =1+\sec ^{2}(x)\left(\tan ^{2}(x)-1\right) \\
& =(-1)^{2}+\sec ^{2}(x) \sum_{n=1}^{2}(-1)^{n+1} \tan ^{4-2 n}(x)
\end{aligned}
$$

We assume that (1.2.1) is true for $k=m, m \geq 1$ and prove that is true for $k=m+1$.

$$
\tan ^{2(m+1)}(x)=\tan ^{2 m}(x) \cdot \tan ^{2}(x)=-\tan ^{2 m}(x)+\tan ^{2 m}(x) \sec ^{2}(x)
$$

For hypotheses induction, we get:

$$
\begin{aligned}
& \tan ^{2(m+1)}(x) \\
& =-\left[(-1)^{m}+\sec ^{2}(x) \sum_{n=1}^{m}(-1)^{n+1} \tan ^{2 m-2 n}(x)\right]+\sec ^{2}(x) \tan ^{2 m}(x) \\
& =(-1)^{m+1}+\sec ^{2}(x) \sum_{n=1}^{m+1}(-1)^{n+1} \tan ^{2(m+1)-2 n}(x)
\end{aligned}
$$


Therefore, the relation is true for all $k, k \geq 1$.

2) Also use mathematical induction on $k, k \geq 1$.

For $k=1$, we find that:

$$
\tan ^{3}(x)=\tan (x)\left(-1+\sec ^{2}(x)\right)=-\tan (x)+\tan (x) \sec ^{2}(x)
$$

For $k=2$, then

$$
\begin{aligned}
\tan ^{5}(x) & =\tan ^{3}(x)\left(-1+\sec ^{2}(x)\right) \\
& =-\tan ^{3}(x)+\tan ^{3}(x) \sec ^{2}(x) \\
& =\tan (x)-\sec ^{2}(x) \tan (x)+\sec ^{2}(x) \tan ^{3}(x) \\
& =\tan (x)+\sec ^{2}(x)\left(\tan ^{3}(x)-\tan (x)\right) \\
& =(-1)^{2} \tan (x)+\sec ^{2}(x) \sum_{n=1}^{2}(-1)^{n+1} \tan ^{5-2 n}(x)
\end{aligned}
$$

We assume that (1.2.2) is true for $k=m, m \geq 1$ and prove that is true for $k=m+1$

$$
\tan ^{2(m+1)+1}(x)=\tan ^{2 m+1}(x) \cdot \tan ^{2}(x)=-\tan ^{2 m+1}(x)+\tan ^{2 m+1}(x) \sec ^{2}(x)
$$

For hypotheses induction, we get:

$$
\begin{aligned}
& \tan ^{2(m+1)+1}(x) \\
& =-\left[(-1)^{m} \tan (x)+\sec ^{2}(x) \sum_{n=1}^{m}(-1)^{n+1} \tan ^{2 m-2 n+1}(x)\right]+\sec ^{2}(x) \tan ^{2 m+1}(x) \\
& =(-1)^{m+1} \tan (x)+\sec ^{2}(x) \sum_{n=1}^{m+1}(-1)^{n+1} \tan ^{2(m+1)+1-2 n}(x)
\end{aligned}
$$

Therefore, the relation is true for all $k, k \geq 1$.

3) We can prove it with the same method.

4) We can prove it with the same method. \#

Theorem 1.3: For all $k \in N$, then:

$$
\begin{aligned}
& \sec ^{2 k}(x)=\sec ^{2}(x) \sum_{n=0}^{k-1}\left(\begin{array}{c}
k-1 \\
n
\end{array}\right) \tan ^{2 n}(x) . \\
& \csc ^{2 k}(x)=\csc ^{2}(x) \sum_{n=0}^{k-1}\left(\begin{array}{c}
k-1 \\
n
\end{array}\right) \cot ^{2 n}(x) .
\end{aligned}
$$

Proof:

1)

$$
\begin{aligned}
\sec ^{2 k}(x) & =\sec ^{2}(x) \cdot\left(\sec ^{2}(x)\right)^{k-1}=\sec ^{2}(x) \cdot\left(1+\tan ^{2}(x)\right)^{k-1} \\
& =\sec ^{2}(x) \sum_{n=0}^{k-1}\left(\begin{array}{c}
k-1 \\
n
\end{array}\right) \tan ^{2 n}(x)
\end{aligned}
$$

(by binomial

theory).

2)

$$
\begin{aligned}
\csc ^{2 k}(x) & =\csc ^{2}(x) \cdot\left(\csc ^{2}(x)\right)^{k-1}=\csc ^{2}(x) \cdot\left(1+\cot ^{2}(x)\right)^{k-1} \\
& =\csc ^{2}(x) \sum_{n=0}^{k-1}\left(\begin{array}{c}
k-1 \\
n
\end{array}\right) \cot ^{2 n}(x)
\end{aligned}
$$

(by binomial

theory).

\section{The Integration of Trigonometric Function Power}

In this section, we find the integrations of power of function trigonometric 
Theorem 2.1: For all $k \in N$, then:

1) $\int \sin ^{2 k-1}(x) \mathrm{d} x=\frac{(-1)^{k-1}}{2^{2 k-2}} \sum_{n=1}^{k} \frac{(-1)^{n-1}\left(\begin{array}{c}2 k-1 \\ n-1\end{array}\right)}{2 k-2 n+1} \cos (2 k-2 n+1) x+c$

2) $\int \sin ^{2 k}(x) \mathrm{d} x=\frac{1}{2^{2 k}}\left(\begin{array}{c}2 k \\ k\end{array}\right) x+\frac{(-1)^{k}}{2^{2 k-1}} \sum_{n=0}^{k-1} \frac{(-1)^{n}\left(\begin{array}{c}2 k \\ n\end{array}\right)}{2 k-2 n} \sin (2 k-2 n) x+c$

3) $\int \cos ^{2 k-1}(x) \mathrm{d} x=\frac{1}{2^{2 k-2}} \sum_{n=1}^{k} \frac{\left(\begin{array}{c}2 k-1 \\ n-1\end{array}\right)}{2 k-2 n+1} \sin (2 k-2 n+1) x+c$.

4) $\int \cos ^{2 k}(x) \mathrm{d} x=\frac{1}{2^{2 k}}\left(\begin{array}{c}2 k \\ k\end{array}\right) x+\frac{1}{2^{2 k-1}} \sum_{n=0}^{k-1} \frac{\left(\begin{array}{c}2 k \\ n\end{array}\right)}{2 k-2 n} \sin (2 k-2 n) x+c . \#$

Proof: Directed from Theorem (1.1). \#

Theorem 2.2: For all $k \in N$, then:

1) $\int \tan ^{2 k}(x) \mathrm{d} x=(-1)^{k} x+\sum_{n=1}^{k} \frac{(-1)^{n+1}}{2 k-2 n+1} \tan ^{2 k-2 n+1}(x)+c$.

2) $\int \tan ^{2 k+1}(x) \mathrm{d} x=(-1)^{k+1} \ln |\cos (x)|+\sum_{n=1}^{k} \frac{(-1)^{n+1}}{2 k-2 n+2} \tan ^{2 k-2 n+2}(x)+c$

3) $\int \cot ^{2 k}(x) \mathrm{d} x=(-1)^{k} x+\sum_{n=1}^{k} \frac{(-1)^{n}}{2 k-2 n+1} \cot ^{2 k-2 n+1}(x)+c$.

4) $\int \cot ^{2 k+1}(x) \mathrm{d} x=(-1)^{k} \ln |\sin (x)|+\sum_{n=1}^{k} \frac{(-1)^{n}}{2 k-2 n+2} \cot ^{2 k-2 n+2}(x)+c$

5) $\int \sec ^{2 k}(x) \mathrm{d} x=\sum_{n=0}^{k-1} \frac{\left(\begin{array}{c}k-1 \\ n\end{array}\right)}{2 n+1} \tan ^{2 n+1}(x)+c$.

6) $\int \csc ^{2 k}(x) \mathrm{d} x=-\sum_{n=0}^{k-1} \frac{\left(\begin{array}{c}k-1 \\ n\end{array}\right)}{2 n+1} \cot ^{2 n+1}(x)+c$.

Proof: Directed from Theorem (1.2) and Theorem (1.3).\#

Lemma 2.3: For all $k \in N$, then:

$$
\int \sec ^{2 k+1}(x) \mathrm{d} x=\frac{1}{2 k}\left[\sec ^{2 k-1}(x) \tan (x)+(2 k-1) \int \sec ^{2 k-1}(x) \mathrm{d} x\right]
$$

Proof: $\int \sec ^{2 k+1}(x) \mathrm{d} x=\int \sec ^{2 k-1}(x) \sec ^{2}(x) \mathrm{d} x$

Using integration by parts:

Let $u=\sec ^{2 k-1}(x)$, then $\mathrm{d} u=(2 k-1) \sec ^{2 k-1}(x) \tan (x) \mathrm{d} x$

$\mathrm{d} v=\sec ^{2}(x) \mathrm{d} x$, then $v=\tan (x)$

$$
\begin{aligned}
& \therefore \int \sec ^{2 k+1}(x) \mathrm{d} x \\
& =\sec ^{2 k-1}(x) \tan (x)-(2 k-1) \int \sec ^{2 k-1}(x) \tan ^{2}(x) \mathrm{d} x \\
& =\sec ^{2 k-1}(x) \tan (x)-(2 k-1) \int \sec ^{2 k-1}(x)\left(\sec ^{2}(x)-1\right) \mathrm{d} x \\
& =\sec ^{2 k-1}(x) \tan (x)-(2 k-1) \int \sec ^{2 k+1}(x) \mathrm{d} x+(2 k-1) \int \sec ^{2 k-1}(x) \mathrm{d} x
\end{aligned}
$$




$$
\therefore 2 k \int \sec ^{2 k+1}(x) \mathrm{d} x=\sec ^{2 k-1}(x) \tan (x)+(2 k-1) \int \sec ^{2 k-1}(x) \mathrm{d} x .
$$

Hence

$$
\int \sec ^{2 k+1}(x) \mathrm{d} x=\frac{1}{2 k}\left[\sec ^{2 k-1}(x) \tan (x)+(2 k-1) \int \sec ^{2 k-1}(x) \mathrm{d} x\right] . \#
$$

Note: From clearly that:

$$
\int \sec ^{3}(x) \mathrm{d} x=\frac{1}{2}[\sec (x) \tan (x)+\ln |\sec (x)+\tan (x)|]+c .
$$

Theorem 2.4: For all $k \in N$, then:

$$
\begin{aligned}
\int \sec ^{2 k+1}(x) \mathrm{d} x= & \frac{\prod_{i=1}^{k-1}(2 i+1)}{\prod_{i=1}^{k}(2 i)} \ln |\sec (x)+\tan (x)|+\frac{1}{2 k} \sec ^{2 k-1}(x) \tan (x) \\
& +\tan (x) \sum_{j=1}^{k-1} \frac{\prod_{i=j}^{k-1}(2 i+1)}{\prod_{i=j}^{k}(2 i)} \sec ^{2 i-1}(x)+c
\end{aligned}
$$

Proof: Since

$$
\int \sec ^{2 k+1}(x) \mathrm{d} x=\frac{1}{2 k}\left[\sec ^{2 k-1}(x) \tan (x)+(2 k-1) \int \sec ^{2 k-1}(x) \mathrm{d} x\right]
$$

(by Lemma 2.3). Then

$$
\begin{aligned}
& \qquad \int \sec ^{2 k-1}(x) \mathrm{d} x=\frac{1}{2 k-2}\left[\sec ^{2 k-3}(x) \tan (x)+(2 k-3) \int \sec ^{2 k-3}(x) \mathrm{d} x\right] \\
& \text { and } \int \sec ^{2 k-3}(x) \mathrm{d} x=\frac{1}{2 k-4}\left[\sec ^{2 k-5}(x) \tan (x)+(2 k-5) \int \sec ^{2 k-5}(x) \mathrm{d} x\right] . \\
& \text { and so on }
\end{aligned}
$$

$$
\begin{gathered}
\quad \int \sec ^{5}(x) \mathrm{d} x=\frac{1}{4}\left[\sec ^{3}(x) \tan (x)+3 \int \sec ^{3}(x) \mathrm{d} x\right] \\
\text { and } \int \sec ^{3}(x) \mathrm{d} x=\frac{1}{2}[\sec (x) \tan (x)+\ln |\sec (x)+\tan (x)|]+c . \\
\therefore \int \sec ^{2 k+1}(x) \mathrm{d} x \\
=\frac{1}{2 k} \sec ^{2 k-1}(x) \tan (x)+\frac{2 k-1}{2 k(2 k-2)} \sec ^{2 k-3}(x) \tan (x) \\
+\frac{(2 k-1)(2 k-3)}{2 k(2 k-2)(2 k-4)} \sec ^{2 k-5}(x) \tan (x) \\
+\frac{(2 k-1)(2 k-3)(2 k-5)}{2 k(2 k-2)(2 k-4)(2 k-6)} \sec { }^{2 k-7}(x) \tan (x) \\
+\frac{(2 k-1)(2 k-3)(2 k-5)(2 k-7)}{2 k(2 k-2)(2 k-4)(2 k-6)(2 k-8)} \sec ^{2 k-9}(x) \tan (x)
\end{gathered}
$$




$$
\begin{gathered}
+\cdots \\
+\frac{(2 k-1)(2 k-3)(2 k-5)(2 k-7) \cdots(5)}{2 k(2 k-2)(2 k-4)(2 k-6)(2 k-8) \cdots(4)} \sec ^{3}(x) \tan (x) \\
+\frac{(2 k-1)(2 k-3)(2 k-5)(2 k-7) \cdots(5)(3)}{2 k(2 k-2)(2 k-4)(2 k-6)(2 k-8) \cdots(4)(2)} \sec (x) \tan (x) \\
+\frac{(2 k-1)(2 k-3)(2 k-5)(2 k-7) \cdots(5)(3)}{2 k(2 k-2)(2 k-4)(2 k-6)(2 k-8) \cdots(4)(2)} \ln |\sec (x)+\tan (x)|+c \\
\therefore \int \sec ^{2 k+1}(x) \mathrm{d} x=\frac{\prod_{i=1}^{k-1}(2 i+1)}{\prod_{i=1}^{k}(2 i)} \ln |\sec (x)+\tan (x)|+\frac{1}{2 k} \sec ^{2 k-1}(x) \tan (x) \\
+\tan (x) \sum_{j=1}^{k-1} \frac{\prod_{i=j}^{k-1}(2 i+1)}{\prod_{i=j}^{k}(2 i)} \sec ^{2 i-1}(x)+c
\end{gathered}
$$

Lemma 2.5: For all $k \in N$, then:

$$
\int \csc ^{2 k+1}(x) \mathrm{d} x=\frac{1}{2 k}\left[-\csc ^{2 k-1}(x) \cot (x)+(2 k-1) \int \csc ^{2 k-1}(x) \mathrm{d} x\right]
$$

Proof: $\int \csc ^{2 k+1}(x) \mathrm{d} x=\int \csc ^{2 k-1}(x) \csc ^{2}(x) \mathrm{d} x$

Using integration by parts:

Let $u=\csc ^{2 k-1}(x)$, then $\mathrm{d} u=-(2 k-1) \csc ^{2 k-1}(x) \cot (x) \mathrm{d} x$

$\mathrm{d} v=\csc ^{2}(x) \mathrm{d} x$, then $v=-\cot (x)$

$\therefore \int \csc ^{2 k+1}(x) \mathrm{d} x$

$=-\csc ^{2 k-1}(x) \cot (x)-(2 k-1) \int \csc ^{2 k-1}(x) \cot ^{2}(x) \mathrm{d} x$

$=-\csc ^{2 k-1}(x) \cot (x)-(2 k-1) \int \csc ^{2 k-1}(x)\left(\csc ^{2}(x)-1\right) \mathrm{d} x$

$=-\csc ^{2 k-1}(x) \cot (x)-(2 k-1) \int \csc ^{2 k+1}(x) \mathrm{d} x+(2 k-1) \int \csc ^{2 k-1}(x) \mathrm{d} x$

$\therefore 2 k \int \csc ^{2 k+1}(x) \mathrm{d} x=-\csc ^{2 k-1}(x) \cot (x)+(2 k-1) \int \csc ^{2 k-1}(x) \mathrm{d} x$

$\therefore \int \csc ^{2 k+1}(x) \mathrm{d} x=\frac{1}{2 k}\left[-\csc ^{2 k-1}(x) \tan (x)+(2 k-1) \int \csc ^{2 k-1}(x) \mathrm{d} x\right]$.

Note: From clearly that:

$\int \csc ^{3}(x) \mathrm{d} x=-\frac{1}{2}[\csc (x) \cot (x)+\ln |\csc (x)+\cot (x)|]+c$.

Theorem 2.6: For all $k \in N$, then:

$$
\begin{aligned}
\int \csc ^{2 k+1}(x) \mathrm{d} x= & -\frac{\prod_{i=1}^{k-1}(2 i+1)}{\prod_{i=1}^{k}(2 i)} \ln |\csc (x)+\cot (x)|-\frac{1}{2 k} \csc ^{2 k-1}(x) \cot (x) \\
& -\cot (x) \sum_{j=1}^{k-1} \frac{\prod_{i=j}^{k-1}(2 i+1)}{\prod_{i=j}^{k}(2 i)} \csc ^{2 i-1}(x)+c
\end{aligned}
$$

Proof: Since 
$\int \csc ^{2 k+1}(x) \mathrm{d} x=\frac{1}{2 k}\left[-\csc ^{2 k-1}(x) \cot (x)+(2 k-1) \int \csc ^{2 k-1}(x) \mathrm{d} x\right] \quad$ (by Lemma

2.3) Then

$$
\begin{gathered}
\int \csc ^{2 k-1}(x) \mathrm{d} x=\frac{1}{2 k-2}\left[-\csc ^{2 k-3}(x) \cot (x)+(2 k-3) \int \csc ^{2 k-3}(x) \mathrm{d} x\right] \\
\text { and } \int \csc ^{2 k-3}(x) \mathrm{d} x=\frac{1}{2 k-4}\left[-\csc ^{2 k-5}(x) \cot (x)+(2 k-5) \int \csc ^{2 k-5}(x) \mathrm{d} x\right] .
\end{gathered}
$$$$
\text { ... }
$$

and so on

$$
\int \csc ^{5}(x) \mathrm{d} x=\frac{1}{4}\left[-\csc ^{3}(x) \cot (x)+3 \int \csc ^{3}(x) \mathrm{d} x\right]
$$

and $\int \csc ^{3}(x) \mathrm{d} x=\frac{1}{2}[-\csc (x) \cot (x)-\ln |\csc (x)+\cot (x)|]+c$.

$$
\begin{aligned}
& \therefore \int \csc ^{2 k+1}(x) \mathrm{d} x \\
& =\frac{-1}{2 k} \csc ^{2 k-1}(x) \cot (x)-\frac{2 k-1}{2 k(2 k-2)} \csc ^{2 k-3}(x) \cot (x) \\
& -\frac{(2 k-1)(2 k-3)}{2 k(2 k-2)(2 k-4)} \csc ^{2 k-5}(x) \cot (x) \\
& -\frac{(2 k-1)(2 k-3)(2 k-5)}{2 k(2 k-2)(2 k-4)(2 k-6)} \csc ^{2 k-7}(x) \cot (x) \\
& -\frac{(2 k-1)(2 k-3)(2 k-5)(2 k-7)}{2 k(2 k-2)(2 k-4)(2 k-6)(2 k-8)} \csc ^{2 k-9}(x) \cot (x) \\
& -\frac{(2 k-1)(2 k-3)(2 k-5)(2 k-7) \cdots(5)}{2 k(2 k-2)(2 k-4)(2 k-6)(2 k-8) \cdots(4)} \csc ^{3}(x) \cot (x) \\
& -\frac{(2 k-1)(2 k-3)(2 k-5)(2 k-7) \cdots(5)(3)}{2 k(2 k-2)(2 k-4)(2 k-6)(2 k-8) \cdots(4)(2)} \csc (x) \cot (x) \\
& -\frac{(2 k-1)(2 k-3)(2 k-5)(2 k-7) \cdots(5)(3)}{2 k(2 k-2)(2 k-4)(2 k-6)(2 k-8) \cdots(4)(2)} \ln |\csc (x)+\cot (x)|+c \\
& \therefore \int \csc ^{2 k+1}(x) \mathrm{d} x=-\frac{\prod_{i=1}^{k-1}(2 i+1)}{\prod_{i=1}^{k}(2 i)} \ln |\csc (x)+\cot (x)|-\frac{1}{2 k} \csc ^{2 k-1}(x) \cot (x) \\
& -\cot (x) \sum_{j=1}^{k-1} \frac{\prod_{i=j}^{k-1}(2 i+1)}{\prod_{i=j}^{k}(2 i)} \csc ^{2 i-1}(x)+c
\end{aligned}
$$

\section{The Integration of Multiply Trigonometric Function Power}

Theorem 3.1: For all $n \in N$, then:

$$
\text { 1) } \int \sin ^{n}(x) \cos (x) \mathrm{d} x=\left\{\begin{array}{ll}
\frac{1}{n+1} \sin ^{n+1}(x)+c, & n \neq-1 \\
\ln |\sin (x)|+c, & n=-1
\end{array}\right. \text {. }
$$


2) $\int \cos ^{n}(x) \sin (x) \mathrm{d} x=\left\{\begin{array}{ll}\frac{-1}{n+1} \cos ^{n+1}(x)+c, & n \neq-1 \\ -\ln |\cos (x)|+c, & n=-1\end{array}\right.$.

3) $\int \tan ^{n}(x) \sec ^{2}(x) \mathrm{d} x=\left\{\begin{array}{ll}\frac{1}{n+1} \tan ^{n+1}(x)+c, & n \neq-1 \\ \ln |\tan (x)|+c, & n=-1\end{array}\right.$.

4) $\int \cot ^{n}(x) \csc ^{2}(x) \mathrm{d} x=\left\{\begin{array}{ll}\frac{-1}{n+1} \cot ^{n+1}(x)+c, & n \neq-1 \\ -\ln |\cot (x)|+c, & n=-1\end{array}\right.$.

5) $\int \sec ^{n}(x) \tan (x) \mathrm{d} x=\left\{\begin{array}{ll}\frac{1}{n} \sec ^{n}(x)+c, & n \neq-1 \\ -\cos (x)+c, & n=-1\end{array}\right.$.

6) $\int \csc ^{n}(x) \cot (x) \mathrm{d} x=\left\{\begin{array}{ll}\frac{-1}{n} \csc ^{n}(x)+c, & n \neq-1 \\ \sin (x)+c, & n=-1\end{array}\right.$.

where $c$ is integral constant.

Proof: Directed proof. \#

Theorem 3.2: For all $n, m \in N$ and $m$ is an odd number, then:

$\int \sin ^{m}(x) \cos ^{n}(x) \mathrm{d} x=\sum_{i=0}^{k} \frac{(-1)^{i+1}}{n+1+2 i}\left(\begin{array}{l}k \\ i\end{array}\right) \cos ^{n+1+2 i}(x)+c$.

Proof: Since $m$ is an odd number, then $\exists k \in N$ such that $m=2 k+1$.

$\int \sin ^{m}(x) \cos ^{n}(x) \mathrm{d} x$

$=\int \sin ^{2 k+1}(x) \cos ^{n}(x) \mathrm{d} x=\int\left(\sin ^{2}(x)\right)^{k} \sin (x) \cos ^{n}(x) \mathrm{d} x$

$=\int\left(1-\cos ^{2}(x)\right)^{k} \sin (x) \cos ^{n}(x) \mathrm{d} x=\int \sum_{i=0}^{k}(-1)^{i}\left(\begin{array}{l}k \\ i\end{array}\right) \cos ^{2 i}(x) \sin (x) \cos ^{n}(x) \mathrm{d} x$

$=\sum_{i=0}^{k}(-1)^{i}\left(\begin{array}{l}k \\ i\end{array}\right) \int \cos ^{n+2 i}(x) \sin (x) d x=\sum_{i=0}^{k} \frac{(-1)^{i+1}}{n+1+2 i}\left(\begin{array}{l}k \\ i\end{array}\right) \cos ^{n+1+2 i}(x)+c \quad \#$

Theorem 3.3: For all $n, m \in N$ and $m$ is an even number, then:

$$
\begin{aligned}
& \int \sin ^{m}(x) \cos ^{n}(x) \mathrm{d} x \\
& =\left\{\begin{array}{c}
\sum_{i=0}^{k}(-1)^{i}\left(\begin{array}{l}
k \\
i
\end{array}\right)\left[\frac{1}{2^{\alpha}}\left(\begin{array}{c}
\alpha \\
\frac{\alpha}{2}
\end{array}\right) x+\frac{1}{2^{\alpha-1}} \sum_{j=0}^{\frac{\alpha}{2}-1} \frac{\left(\begin{array}{c}
\alpha \\
j
\end{array}\right)}{\alpha-2 j} \sin (\alpha-2 j) x\right]+c \\
\text { if } \alpha=2 i+n, n \text { is even }
\end{array}\right] \\
& \sum_{i=0}^{k}(-1)^{i}\left(\begin{array}{c}
k \\
i
\end{array}\right)\left[\begin{array}{c}
\left.\frac{1}{2^{\alpha-1}} \sum_{j=1}^{\frac{\alpha+1}{2}} \frac{\left(\begin{array}{c}
\alpha-1 \\
j-1
\end{array}\right)}{\alpha-2 j+1} \sin (\alpha-2 j+1) x\right]+c \\
\text { if } \alpha=2 i+n, n \text { is odd }
\end{array}\right.
\end{aligned}
$$


Proof: Since $m$ is an even number, then $\exists k \in N$ such that $m=2 k$.

$$
\begin{aligned}
& \int \sin ^{m}(x) \cos ^{n}(x) \mathrm{d} x \\
& =\int \sin ^{2 k}(x) \cos ^{n}(x) \mathrm{d} x \\
& =\int\left(\sin ^{2}(x)\right)^{k} \cos ^{n}(x) \mathrm{d} x \\
& =\int\left(1-\cos ^{2}(x)\right)^{k} \cos ^{n}(x) \mathrm{d} x \\
& =\int \sum_{i=0}^{k}(-1)^{i}\left(\begin{array}{c}
k \\
i
\end{array}\right) \cos ^{2 i+n}(x) \mathrm{d} x
\end{aligned}
$$

By Theorem 2.1, we have (3.3).

Theorem 3.4: For all $n, m \in N$ and $m$ is an odd number, then:

$$
\int \tan ^{m}(x) \sec ^{n}(x) \mathrm{d} x=\sum_{i=0}^{k} \frac{(-1)^{k+i}}{n+2 i}\left(\begin{array}{l}
k \\
i
\end{array}\right) \sec ^{n+2 i}(x)+c .
$$

Proof: Since $m$ is an odd number, then $\exists k \in N$ such that $m=2 k+1$.

$$
\begin{aligned}
& \int \tan ^{m}(x) \sec ^{n}(x) \mathrm{d} x \\
& =\int \tan ^{2 k+1}(x) \sec ^{n}(x) \mathrm{d} x=\int\left(\tan ^{2}(x)\right)^{k} \tan (x) \sec ^{n}(x) \mathrm{d} x \\
& =\int\left(\sec ^{2}(x)-1\right)^{k} \tan (x) \sec ^{n}(x) \mathrm{d} x \\
& =\int(-1)^{k}\left(1-\sec ^{2}(x)\right)^{k} \tan (x) \sec ^{n}(x) \mathrm{d} x \\
& =\int(-1)^{k} \sum_{i=0}^{k}(-1)^{i}\left(\begin{array}{c}
k \\
i
\end{array}\right) \sec ^{2 i}(x) \tan (x) \sec ^{n}(x) \mathrm{d} x \\
& =\sum_{i=0}^{k}(-1)^{k+i}\left(\begin{array}{c}
k \\
i
\end{array}\right) \int \sec ^{n+2 i-1}(x) \sec (x) \tan (x) \mathrm{d} x \\
& =\sum_{i=0}^{k} \frac{(-1)^{k+i}}{n+2 i}\left(\begin{array}{c}
k \\
i
\end{array}\right) \sec ^{n+2 i}(x)+c
\end{aligned}
$$

Theorem 3.5: For all $n, m \in N$ and $m$ is an even number, then:

$$
\begin{aligned}
& \int \tan ^{m}(x) \sec ^{n}(x) d x= \\
& \int \tan ^{m}(x) \sec ^{n}(x) \mathrm{d} x
\end{aligned}
$$

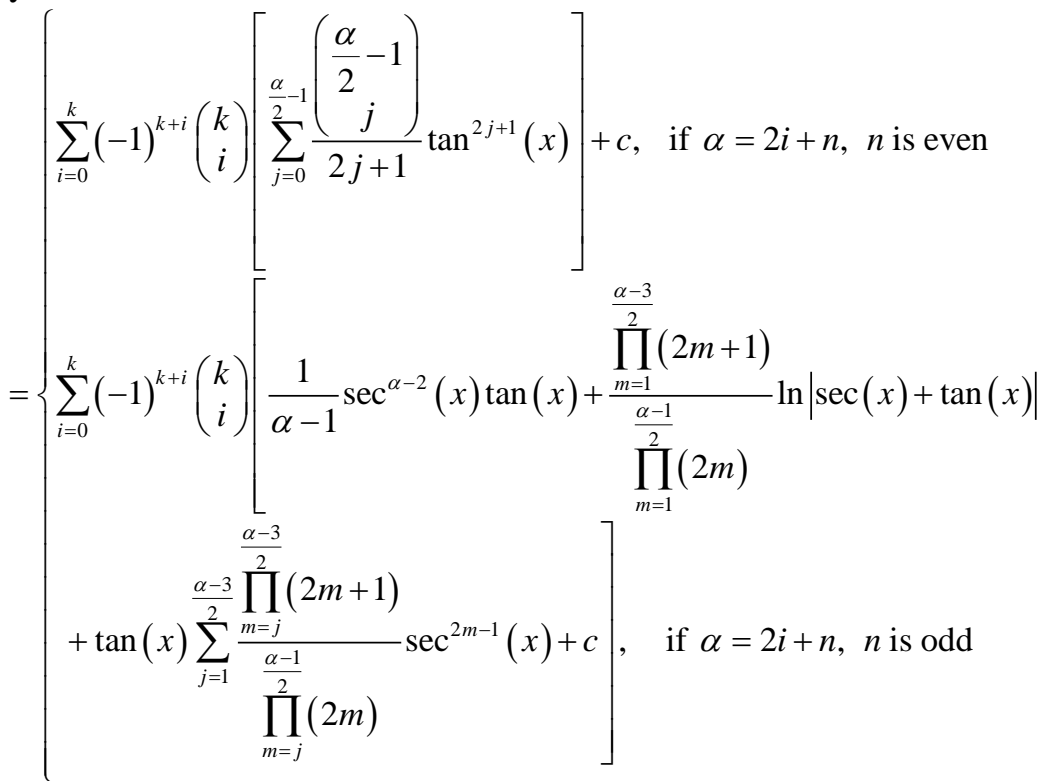


Proof: Since $m$ is an even number, then $\exists k \in N$ such that $m=2 k$.

$$
\begin{aligned}
& \int \tan ^{m}(x) \sec ^{n}(x) \mathrm{d} x \\
& =\int \tan ^{2 k}(x) \sec ^{n}(x) \mathrm{d} x=\int\left(\tan ^{2}(x)\right)^{k} \sec ^{n}(x) \mathrm{d} x \\
& =\int\left(\sec ^{2}(x)-1\right)^{k} \sec ^{n}(x) \mathrm{d} x=\int(-1)^{k}\left(1-\sec ^{2}(x)\right)^{k} \sec ^{n}(x) \mathrm{d} x \\
& =\int \sum_{i=0}^{k}(-1)^{k+i}\left(\begin{array}{c}
k \\
i
\end{array}\right) \sec ^{2 i+n}(x) \mathrm{d} x
\end{aligned}
$$

By Theorem 2.2, we have (3.5). \#

Theorem 3.6: For all $n, m \in N$ and $m$ is an odd number, then:

$\int \cot ^{m}(x) \csc ^{n}(x) \mathrm{d} x=\sum_{i=0}^{k} \frac{(-1)^{k+i+1}}{n+2 i}\left(\begin{array}{l}k \\ i\end{array}\right) \csc ^{n+2 i}(x)+c$.

Proof: Since $m$ is an odd number, then $\exists k \in N$ such that $m=2 k+1$.

$$
\begin{aligned}
& \int \cot ^{m}(x) \csc ^{n}(x) \mathrm{d} x \\
& =\int \cot ^{2 k+1}(x) \csc ^{n}(x) \mathrm{d} x=\int \cot ^{2 k}(x) \cot (x) \csc ^{n}(x) \mathrm{d} x \\
& =\int\left(\cot ^{2}(x)\right)^{k} \cot (x) \csc ^{n}(x) \mathrm{d} x=\int\left(\csc ^{2}(x)-1\right)^{k} \cot (x) \csc ^{n}(x) \mathrm{d} x \\
& =\int(-1)^{k}\left(1-\csc ^{2}(x)\right)^{k} \cot (x) \csc ^{n}(x) \mathrm{d} x \\
& =\int(-1)^{k} \sum_{i=0}^{k}(-1)^{i}\left(\begin{array}{c}
k \\
i
\end{array}\right) \csc ^{2 i}(x) \cot (x) \csc ^{n}(x) \mathrm{d} x \\
& =\sum_{i=0}^{k}-(-1)^{k+i}\left(\begin{array}{c}
k \\
i
\end{array}\right) \int \csc ^{n+2 i-1}(x)(-\csc (x)) \cot (x) \mathrm{d} x \\
& =\sum_{i=0}^{k} \frac{(-1)^{k+i+1}}{n+2 i}\left(\begin{array}{c}
k \\
i
\end{array}\right) \csc ^{n+2 i}(x)+c
\end{aligned}
$$

Theorem 3.7: For all $n, m \in N$ and $m$ is an even number, then: $\int \cot ^{m}(x) \csc ^{n}(x) d x=$ $\int \cot ^{m}(x) \csc ^{n}(x) \mathrm{d} x$

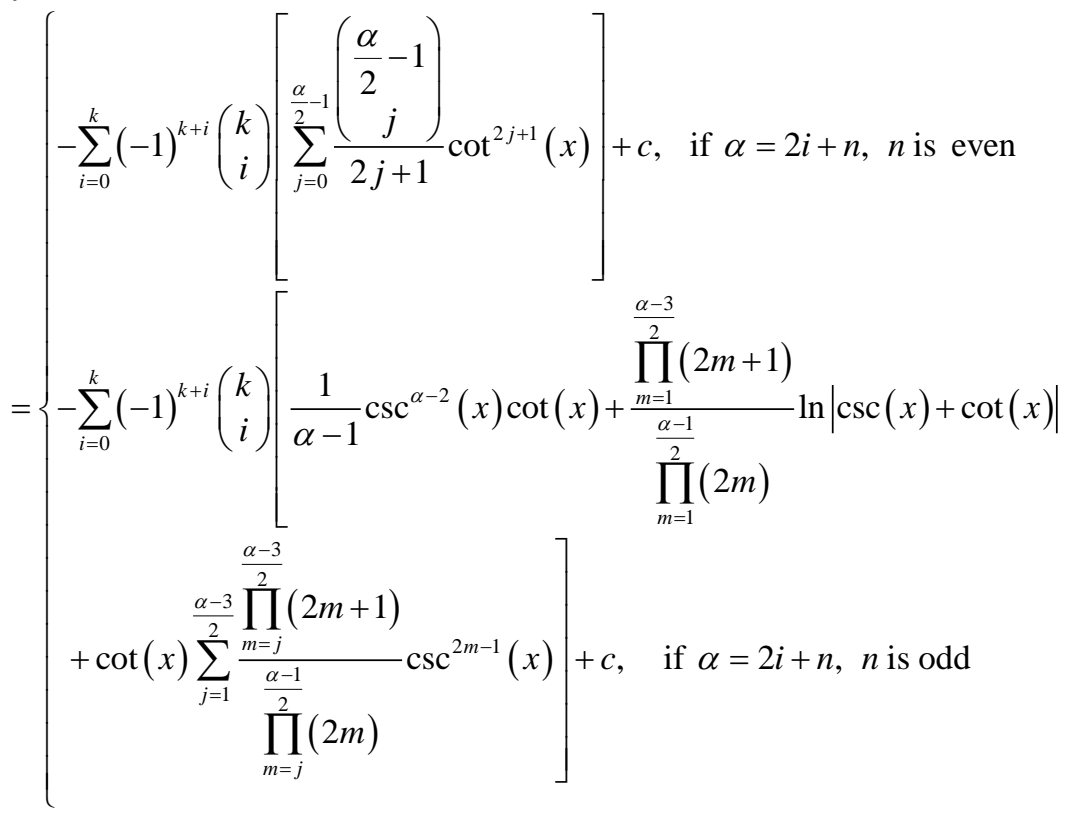


Proof: Since $m$ is an even number, then $\exists k \in N$ such that $m=2 k$

$$
\begin{aligned}
& \int \cot ^{m}(x) \csc ^{n}(x) \mathrm{d} x \\
& =\int \cot ^{2 k}(x) \csc ^{n}(x) \mathrm{d} x \\
& =\int\left(\cot ^{2}(x)\right)^{k} \csc ^{n}(x) \mathrm{d} x \\
& =\int\left(\csc ^{2}(x)-1\right)^{k} \csc ^{n}(x) \mathrm{d} x \\
& =\int(-1)^{k} \sum_{i=0}^{k}(-1)^{i}\left(\begin{array}{l}
k \\
i
\end{array}\right) \csc ^{2 i+n}(x) \mathrm{d} x \\
& =\int \sum_{i=0}^{k}(-1)^{k+i}\left(\begin{array}{l}
k \\
i
\end{array}\right) \csc ^{2 i+n}(x) \mathrm{d} x \\
& =\sum_{i=0}^{k}(-1)^{k+i}\left(\begin{array}{l}
k \\
i
\end{array}\right) \int \csc ^{2 i+n}(x) \mathrm{d} x
\end{aligned}
$$

Using Theorems 2.2 and 2.5, we have (3.7). \#

Remark: There are many integration formulas, we can be finding it by previous results which obtained it from this paper. For example:

1) $\int \sin ^{m}(x) f(x) \mathrm{d} x, \int \cos ^{m}(x) f(x) \mathrm{d} x$,

where $f(x) \in\left\{\tan ^{n}(x), \cot ^{n}(x), \sec ^{n}(x), \csc ^{n}(x)\right\}, \forall n, m \in N$.

2) $\int \tan ^{m}(x) g(x) \mathrm{d} x$, where $g(x) \in\left\{\cot ^{n}(x), \sec ^{n}(x), \csc ^{n}(x)\right\}$, $\forall n, m \in N$.

3) $\int \cot ^{m}(x) \sec ^{n}(x) \mathrm{d} x$.

\section{The Hyperbolic Functions}

The combinations of the exponential functions $\exp (x)$ and $\exp (-x)$ called hyperbolic functions. These functions which arise in various engineering applications, have many properties in common with the trigonometric functions. The hyperbolic functions have resulted from vibratory motions inside elastic solid and more general in many problems where mechanical energy is gradually absorbed by a surrounding medium. There are importance applications on hyperbolic functions and power integration of hyperbolic functions in physics, mathematics transformations and numerical analysis.

Remark: The proofs for this following result are similarly of the results for previous sections.

\subsection{The Hyperbolic Functions Power}

Theorem 4.1.1: For all $k \in N$, then:

1) $\sinh ^{2 k-1}(x)=\frac{1}{2^{2 k-2}} \sum_{n=0}^{k-1}(-1)^{n}\left(\begin{array}{c}2 k-1 \\ n\end{array}\right) \sinh (2 k-1-2 n) x$.

2) $\sinh ^{2 k}(x)=\frac{(-1)^{k}}{2^{2 k}}\left(\begin{array}{c}2 k \\ k\end{array}\right)+\frac{1}{2^{2 k-1}} \sum_{n=0}^{k-1}(-1)^{n}\left(\begin{array}{c}2 k \\ n\end{array}\right) \cosh (2 k-2 n) x$.

3) $\cosh ^{2 k-1}(x)=\frac{1}{2^{2 k-2}} \sum_{n=0}^{k-1}\left(\begin{array}{c}2 k-1 \\ n\end{array}\right) \cosh (2 k-1-2 n) x$. 

4) $\cosh ^{2 k}(x)=\frac{1}{2^{2 k}}\left(\begin{array}{c}2 k \\ k\end{array}\right)+\frac{1}{2^{2 k-1}} \sum_{n=0}^{k-1}\left(\begin{array}{c}2 k \\ n\end{array}\right) \cosh (2 k-2 n) x$.
5) $\tanh ^{2 k}(x)=1-\operatorname{sech}^{2}(x) \sum_{n=1}^{k} \tanh ^{2 k-2 n}(x)$.
6) $\tanh ^{2 k+1}(x)=\tanh (x)-\operatorname{sech}^{2}(x) \sum_{n=1}^{k} \tanh ^{2 k-2 n+1}(x)$.
7) $\operatorname{coth}^{2 k}(x)=1+\operatorname{csch}^{2}(x) \sum_{n=1}^{k} \operatorname{coth}^{2 k-2 n}(x)$.
8) $\operatorname{coth}^{2 k+1}(x)=\operatorname{coth}(x)+\operatorname{csch}^{2}(x) \sum_{n=1}^{k} \operatorname{coth}^{2 k-2 n+1}(x)$.
9) $\operatorname{sech}^{2 k}(x)=\operatorname{sech}^{2}(x) \sum_{n=0}^{k-1}(-1)^{n}\left(\begin{array}{c}k-1 \\ n\end{array}\right) \tanh ^{2 n}(x)$.
10) $\operatorname{csch}^{2 k}(x)=\operatorname{csch}^{2}(x) \sum_{n=0}^{k-1}(-1)^{k+n-1}\left(\begin{array}{c}k-1 \\ n\end{array}\right) \operatorname{coth}^{2 n}(x)$.

\subsection{The Integration of Hyperbolic Functions Power}

Theorem 4.2.1: For all $k \in N$, then:

1) $\int \sinh ^{2 k-1}(x)=\frac{1}{2^{2 k-2}} \sum_{n=0}^{k-1} \frac{(-1)^{n}}{2 k-1-2 n}\left(\begin{array}{c}2 k-1 \\ n\end{array}\right) \cosh (2 k-1-2 n) x+c$

2) $\int \sinh ^{2 k}(x)=\frac{(-1)^{k}}{2^{2 k}}\left(\begin{array}{c}2 k \\ n\end{array}\right) x+\frac{1}{2^{2 k-1}} \sum_{n=0}^{k-1} \frac{(-1)^{n}}{2 k-2 n}\left(\begin{array}{c}2 k \\ n\end{array}\right) \sinh (2 k-2 n) x+c$

3) $\int \cosh ^{2 k-1}(x)=\frac{1}{2^{2 k-2}} \sum_{n=0}^{k-1} \frac{1}{2 k-1-2 n}\left(\begin{array}{c}2 k-1 \\ n\end{array}\right) \sinh (2 k-1-2 n) x+c$

4) $\int \cosh ^{2 k}(x)=\frac{1}{2^{2 k}}\left(\begin{array}{c}2 k \\ k\end{array}\right) x+\frac{1}{2^{2 k-1}} \sum_{n=0}^{k-1} \frac{1}{2 k-2 n}\left(\begin{array}{c}2 k \\ n\end{array}\right) \sinh (2 k-2 n) x+c$

5) $\int \tanh ^{2 k}(x)=x-\sum_{n=1}^{k} \frac{1}{2 k+1-2 n} \tanh ^{2 k+1-2 n}(x)+c$.

6) $\int \tanh ^{2 k+1}(x)=\ln |\cosh (x)|-\sum_{n=1}^{k} \frac{1}{2 k+2-2 n} \tanh ^{2 k+2-2 n}(x)+c$.

7) $\int \operatorname{coth}^{2 k}(x)=x-\sum_{n=1}^{k} \frac{1}{2 k+1-2 n} \operatorname{coth}^{2 k+1-2 n}(x)+c$.

8) $\int \operatorname{coth}^{2 k+1}(x)=\ln |\sinh (x)|-\sum_{n=1}^{k} \frac{1}{2 k+2-2 n} \operatorname{coth}^{2 k-2 n+2}(x)+c$.

9) $\int \operatorname{sech}^{2 k}(x)=\sum_{n=0}^{k-1} \frac{(-1)^{n}}{2 n+1}\left(\begin{array}{c}k-1 \\ n\end{array}\right) \tanh ^{2 n+1}(x)+c$.

10) $\int \operatorname{csch}^{2 k}(x)=\sum_{n=0}^{k-1} \frac{(-1)^{k+n}}{2 n+1}\left(\begin{array}{c}k-1 \\ n\end{array}\right) \operatorname{coth}^{2 n+1}(x)+c$.

Lemma 4.2.2: For all $k \in N$, then:

1) $\int \operatorname{sech}^{2 k+1}(x) \mathrm{d} x=\frac{1}{2 k}\left[\operatorname{sech}^{2 k-1}(x) \tanh (x)+(2 k-1) \int \sec ^{2 k-1}(x) \mathrm{d} x\right]$

2) $\int \operatorname{csch}^{2 k+1}(x) \mathrm{d} x=-\frac{1}{2 k}\left[\operatorname{csch}^{2 k-1}(x) \operatorname{coth}(x)+(2 k-1) \int \operatorname{csch}^{2 k-1}(x) \mathrm{d} x\right]$

Theorem 4.2.3: For all $k \in N$, then: 


$$
\begin{aligned}
& \int \sec ^{2 k+1}(x) \mathrm{d} x \\
& \text { 1) }=\frac{\prod_{i=1}^{k-1}(2 i+1)}{\prod_{i=1}^{k}(2 i)} \tan ^{-1} \mathrm{e}^{x}+\frac{1}{2 k} \operatorname{sech}^{2 k-1}(x) \tanh (x) \\
& +\tanh (x) \sum_{j=1}^{k-1} \frac{\prod_{i=j}^{k-1}(2 i+1)}{\prod_{i=j}^{k}(2 i)} \operatorname{sech}^{2 i-1}(x)+c \\
& \int \operatorname{csch}^{2 k+1}(x) \mathrm{d} x \\
& =(-1)^{n+1} \frac{\prod_{i=1}^{k-1}(2 i+1)}{\prod_{i=1}^{k}(2 i)} \ln |\operatorname{csch}(x)+\operatorname{coth}(x)| \\
& \text { 2) } \\
& -\frac{1}{2 k} \operatorname{csch}^{2 k-1}(x) \operatorname{coth}(x) \\
& +\operatorname{coth}(x) \sum_{j=1}^{k-1} \frac{\prod_{i=j}^{k-1}(2 i+1)}{\prod_{i=j}^{k}(2 i)} \operatorname{csch}^{2 i-1}(x)+c
\end{aligned}
$$

\subsection{The Integration of Multiply Trigonometric Function Power}

Theorem 4.3.1: For all $n \in N$, then:

1) $\int \sinh ^{n}(x) \cosh (x) \mathrm{d} x=\left\{\begin{array}{ll}\frac{1}{n+1} \sinh ^{n+1}(x)+c, & n \neq-1 \\ \ln |\sinh (x)|+c, & n=-1\end{array}\right.$.

2) $\int \cosh ^{n}(x) \sinh (x) \mathrm{d} x=\left\{\begin{array}{ll}\frac{1}{n+1} \cosh ^{n+1}(x)+c, & n \neq-1 \\ \ln (\cosh (x))+c, & n=-1\end{array}\right.$.

3) $\int \tanh ^{n}(x) \operatorname{sech}^{2}(x) \mathrm{d} x=\left\{\begin{array}{ll}\frac{1}{n+1} \tanh ^{n+1}(x)+c, & n \neq-1 \\ \ln |\tanh (x)|+c, & n=-1\end{array}\right.$.

4) $\int \operatorname{coth}^{n}(x) \operatorname{csch}^{2}(x) \mathrm{d} x= \begin{cases}\frac{-1}{n+1} \operatorname{coth}^{n+1}(x)+c, & n \neq-1 \\ -\ln |\operatorname{coth}(x)|+c, & n=-1\end{cases}$

5) $\int \operatorname{sech}^{n}(x) \tanh (x) \mathrm{d} x=\left\{\begin{array}{ll}\frac{1}{n} \operatorname{sech}^{n}(x)+c, & n \neq-1 \\ \cosh (x)+c, & n=-1\end{array}\right.$.

6) $\int \operatorname{csch}^{n}(x) \operatorname{coth}(x) \mathrm{d} x=\left\{\begin{array}{ll}\frac{-1}{n} \operatorname{csch}^{n}(x)+c, & n \neq-1 \\ \sinh (x)+c, & n=-1\end{array}\right.$.

where $c$ is integral constant.

Theorem 4.3.2: For all $n, m \in N$ and $m$ is an odd number, then: 
$\int \sinh ^{m}(x) \cosh ^{n}(x) \mathrm{d} x$

1) $=\sum_{i=0}^{k} \frac{(-1)^{k+i}}{n+2 i+1}\left(\begin{array}{l}k \\ i\end{array}\right) \cosh ^{n+2 i+1}(x)+c$

$\int \tanh ^{m}(x) \operatorname{sech}^{n}(x) \mathrm{d} x$

2) $=\sum_{i=0}^{k} \frac{(-1)^{i+1}}{n+2 i}\left(\begin{array}{l}k \\ i\end{array}\right) \operatorname{sech}^{n+2 i}(x)+c$

$\int \operatorname{coth}^{m}(x) \operatorname{csch}^{n}(x) d x$

3) $=-\sum_{i=0}^{k} \frac{1}{n+2 i}\left(\begin{array}{l}k \\ i\end{array}\right) \operatorname{csch}^{n+2 i}(x)+c$

Theorem 4.3.3: For all $n, m \in N$ and $m$ is an even number, then:

$\int \sinh ^{m}(x) \cosh ^{n}(x) \mathrm{d} x$

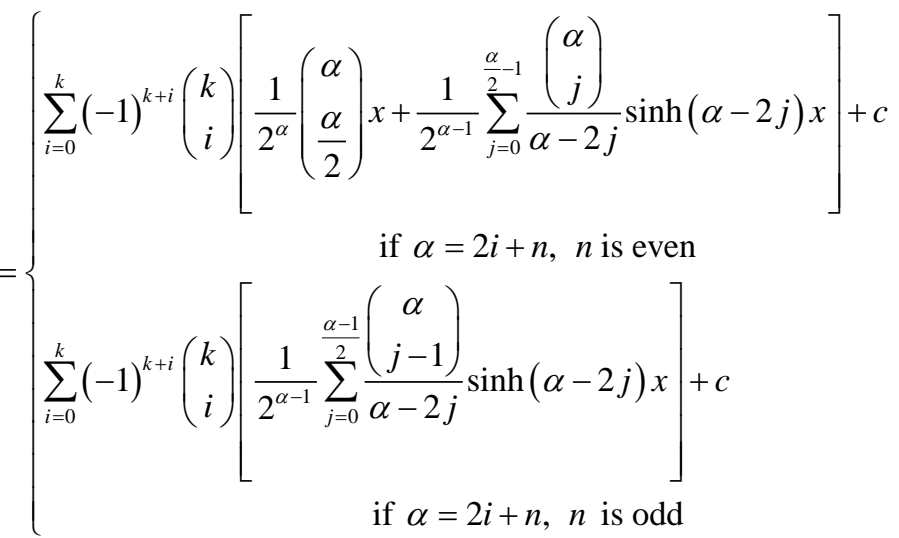

$\int \tanh ^{m}(x) \operatorname{sech}^{n}(x) \mathrm{d} x$

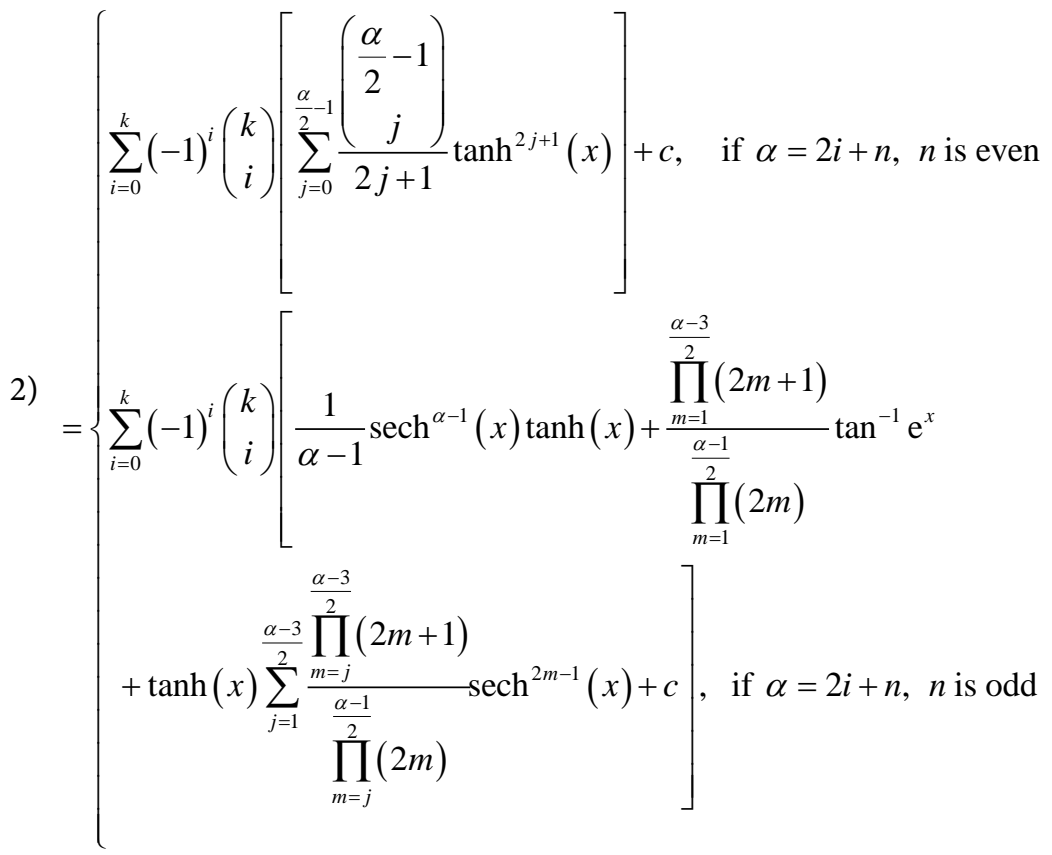




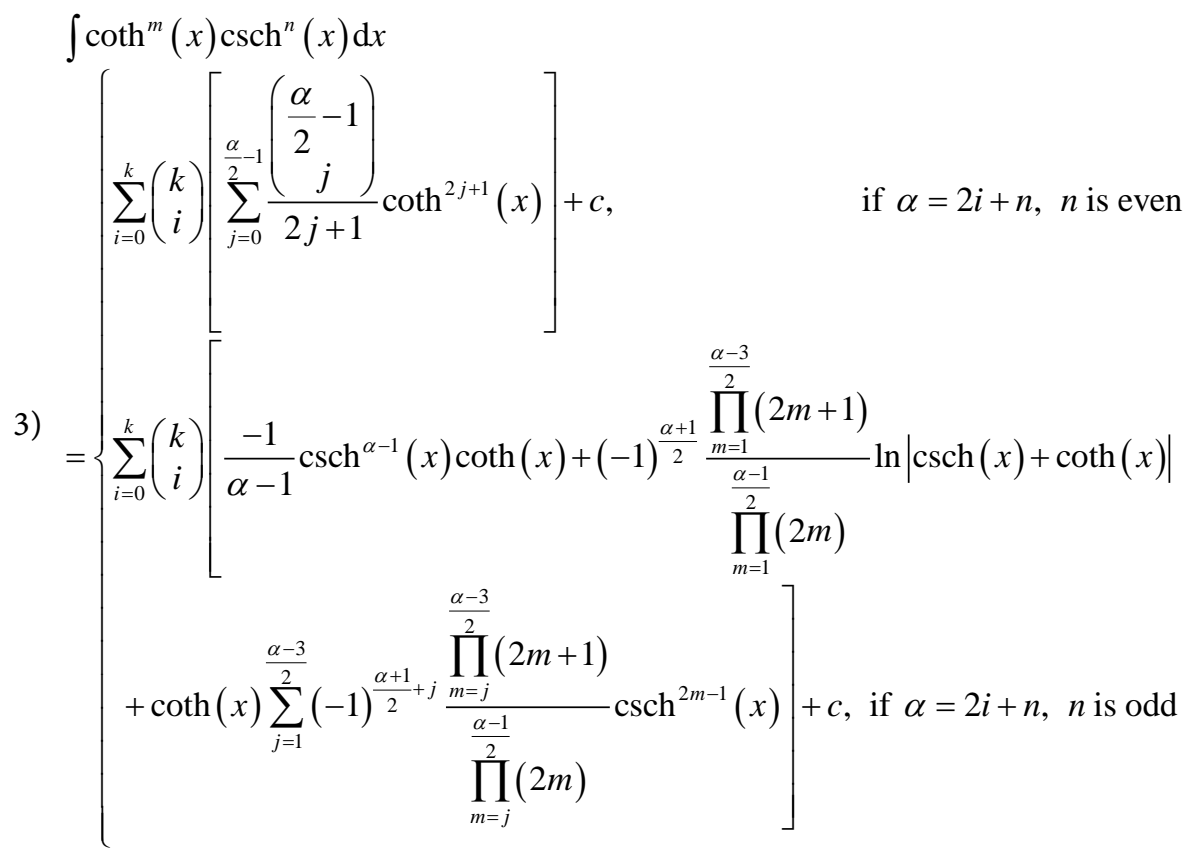

\section{Remark}

There are many integration formulas, we can find it by previous results which obtained it from this paper. For example:

1) $\int \sinh ^{m}(x) f(x) \mathrm{d} x, \int \cosh ^{m}(x) f(x) \mathrm{d} x$, where $f(x) \in\left\{\tanh ^{n}(x), \operatorname{coth}^{n}(x), \operatorname{sech}^{n}(x), \operatorname{csch}^{n}(x)\right\}, \forall n, m \in N$.

2) $\int \tanh ^{m}(x) g(x) \mathrm{d} x$, where

$$
g(x) \in\left\{\operatorname{coth}^{n}(x), \operatorname{sech}^{n}(x), \operatorname{csch}^{n}(x)\right\}, \forall n, m \in N .
$$

3) $\int \operatorname{coth}^{m}(x) \operatorname{sech}^{n}(x) d x$.

\section{Conflicts of Interest}

The authors declare no conflicts of interest regarding the publication of this paper.

\section{References}

[1] Thomas Jr., G.B., Weir, M.D. and Hass, J. (2010) Thomas' Calculus. 12th Edition, Pearson, Boston, New York.

[2] Wilson, F.C. and Adamson, S. (2009) Applied Calculus. Houghton Mifflin Harcourt Publishing Company, Boston, New York.

[3] Wrede, R. and Spiegel, M.R. (2010) Advance Calculus. 3rd Edition, Schaum's Outline Series, New York.

[4] Anton, H., Bivens, L. and Davis, S. (2008) Calculus. 7th Edition, John Wiley and Sons, New York.

[5] Keisler, H.J. (2010) Elementary Calculus. 2nd Edition.

[6] Spiegel, M.R., Lipschutz, S. and Liu, J. (2009) Mathematical Handbook of Formulas and Tables. 3rd Edition, Schaum's Outline Series, New York. 